\title{
Response bias and judgments of the location of clicks in sentences
}

\author{
E. C. DALRYMPLE-ALFORD \\ University of Guelph, Guelph, Ontario, Canada N1G $2 W 1$
}

\begin{abstract}
This study examines the view that response bias is the basis of the "click effect," i.e., the influence of grammatical structure on subjects' location of clicks sounded during the presentation of a sentence. It is argued that, since response bias is more likely to operate when one is unsure of one's perception, if, response bias generates the "click effect," the effect should be weaker for certain than for uncertain responses. Subjects were asked to identify the location of the click and allowed to make more than one response if they were uncertain of their first choice. Using the number of locations selected as an index of uncertainty, it was found that when a subject was less certain, the click was less likely to be judged as having occurred in the major grammatical break. Further, performance was superior when the click had been in the break, and this effect, which was more pronounced for "certain" responses, was not eliminated by correcting for possible response bias. It is concluded that the "click effect" is not attributable solely to response bias.
\end{abstract}

It is generally found that when a click is sounded during auditory presentation of a sentence, a subject is most likely to report its location correctly if it occurs in the major granımatical (i.e., clausal) break, and that when it comes at some other point in the sentence. erroneous judgments of its true location tend towards placing it in the grammatical break, or else in positions adjacent to it (see Fodor. Bever, \& Garrett. 1974, Chap. 6, for a review of the relevant studies). The interpretation of such findings has gone beyond regarding them as demonstrating the "psychological reality" of some accounts of the grammatical structure of sentences. Bever and his associates (cf.. for example. Fodor, Bever, \& Garrett, 1974. pp. 330-333) have maintained that the "click effect " is perceptual in nature and is determined by the way the sentence is segmented during processing. Just how this segmentation causes misperception of the location of the click is left somewhat obscure. To say that clicks "migrate" towards the nearest clause boundary, or that the clausal unit has "relatively high psychological coherence" and therefore "resists interruption" by the click (e.g.. Bever, Lackner, \& Kirk. 1969; Fodor \& Bever, 1965), does not provide more than a figurative redescription of the findings. According to Abrams and Bever (1969). however, the earlier interpretations (e.g., of Fodor \& Bever, 1965) were in terms of a switching of attention from the "speech channel" to the click, this occurring between clauses. the perceptual units of sentence processing. Abrams and Bever suggest instead that "listeners first

This study was supported by Grant A8609 from the National Research Council of Canada. I am indebted to Veronica Hauser for recording the stimuli, collecting the data, and assisting with other stages of the project, and to Harvey Marmurek for comments on an earlier version of the paper. organize the speech into major segments, then they relate the speech and click temporally: it is the latter process that maintains the integrity of the speech units as revealed in the location of clicks" (p. 288). The interpretation of this is not entirely clear to me, but it does seem that errors in locating clicks are still considered to be of perceptual origin.

Reber. on the other hand (Reber, 1973a, b; Reber \& Anderson, 1970), has attributed the tendency to report clicks as occurring in the grammatical break to the effect of linguistic factors on the subject's "attentional priorities and response biases." What he appears to be offering as a single explanation is better considered as two independent, though not necessarily mutually exclusive, accounts. The one in terms of "attentional priorities" is essentially that one "channel" of information can be attended to at any one time, and that "inputs in the attended channel are perceived as occurring earlier in time than are contiguous inputs on the nonattended channel" (Reber \& Anderson, 1970, p. 87). This explanation would still treat the mislocation of clicks as being of perceptual origin. However, Reber's treatment of empirical findings suggests that, beyond its effect through response bias (the other explanation), the position of the major grammatical break has no influence on the subject's placement of the clicks. When response bias is taken into account, all that remains is a general tendency to prepose or postpose the click, a tendency unaffected by the position of the grammatical break.

The only study (cited by Fodor et al., 1974; abstracted in Bever, 1973) that attempts to disprove the response bias interpretation has yet to be reported in sufficient detail to permit evaluation. To date, we are left with Reber's contention that response bias accounts for the "click affect." Reber's case is based 
primarily on two observations: (i) the subject reports the click as having occurred in or adjacent to the grammatical break even when no click had been present (the so-called "subliminal" condition); (ii) when adjustments are made for possible response bias, the superiority of performance when the click was presented in the grammatical break disappears. There is a problem with each of these observations. The distribution of responses in the "subliminal" condition unquestionably reflects response bias, but the magnitude of the bias does not compare with the size of the "click effect" when the click was actually present. In Reber's (1973a) study, the probability of locating the (imaginary) click in the clausal break scarcely exceeded .18 in the "subliminal" condition, whereas it was never less than .55 when the click had actually been presented in the break. The "subliminal" condition may permit the conclusion that "part of the responses [in click studies] may have nothing to do with a subject's perception from the point of view of the way he scans incoming auditory data" (Ladefoged, 1967, p. 161); it does not, on its own, warrant the conclusion that the "click effect" is entirely one of response bias.

Reber's contention that corrections for bias eliminate the "click effect" also has its problems. If we assume that on a given trial the subject is either responding on the basis of his perception or else through response bias, the adjusted proportion of correct responses would $b^{1}\left(p_{c}-p_{b}\right) /\left(1-p_{b}\right)$, where $\mathrm{p}_{c}$ is the observed probability of a correct response, and $\mathrm{pb}_{\mathrm{b}}$ is the probability of a correct response through the operation of response bias. If, for our estimate of $\mathrm{p}_{\mathrm{b}}$, we use $\mathrm{pe}$, the probability of an erroneous response at that location for sentences of identical grammatical structure, the proportion of correct responses adjusted for response bias would be $\left(\mathrm{p}_{\mathrm{c}}-\mathrm{p}_{\mathrm{e}}\right) /\left(1-\mathrm{p}_{\mathrm{e}}\right)$, and not $\mathrm{p}_{\mathrm{c}} /\left(\mathrm{p}_{\mathrm{c}}+\mathrm{p}_{\mathrm{e}}\right)$, which Reber uses, but for which a rationale has not been provided. As far as can be ascertained from Figures 1 and 2 in Reber (1973a), the correction recommended here does not "wash out" the superior performance obtained when the click coincided with the clausal break. It should be noted in any case that corrections based on $\mathrm{p}_{\mathrm{e}}$ must, at least in part, beg the issue by attributing all erroneous responses in the grammatical break to response bias. We may avoid this by estimating $\mathrm{p}_{\mathrm{b}}$ from performance in the "subliminal" condition. Reber's (1973a) results for this condition indicate that estimates of $\mathrm{p}_{b}$ from these would be even smaller; i.e., the correction would have still less effect on the relative superiority of performance when the click and break coincided.

The experiment reported below approaches the question of whether the "click effect" is a perceptual one or the consequence of "response bias" in a different way. The rationale of the study was essentially this: response bias is more likely to operate when one is unsure of one's perceptions, and hence, if the "click effect" is produced by response bias, it should be greater for uncertain than for certain responses; if it is a perceptual effect, it should be weaker for uncertain responses. In this study, the uncertainty of the subject's response was inferred from the number of additional locations he chose to mark when unsure of his first choice. There appear to be only two published studies in which the uncertainty of the subject's response was noted. Fodor and Bever (1965) had subjects indicate their confidence in the correctness of their responses, but only report that "no correlation was discovered between the degree of confidence reported by subjects and the objective accuracy of their performance." Garrett, Bever, and Fodor (1966) had their subjects indicate which responses were guesses, but nothing further was said about this when they reported their findings.

One further comment needs to be made. In previous studies, evidence of the "click effect" had frequently been adduced, not only from the superiority of performance when the click coincided with the clause boundary, and the tendency to erroneously locate the click in these boundaries when in fact it had occurred elsewhere, but also from the tendency to judge the click as having occurred in positions between its true location and the clause boundary. In the absence of an explicit account of how segmentation of a sentence into clauses affects the perception of the click's location, it is doubtful whether responses other than those in the clause boundary should be regarded as supporting evidence.

\section{METHOD}

\section{Materials}

Thirty-six sentences were constructed (each consisting of 12 monosyllabic words), so that an equal number had a major grammatical (i.e.. clausal) break at Positions 5. 9, 13, or 17. each word and "space" being counted as a position (i.e.. there were 25 positions in each sentence. including that before the first and after the last word). The following are examples of the four "types" of sentences, the grammatical break being marked with an asterisk:

(Position 5): Send Tom * since he has a car that is not too small. (Position 9): When we fourd it * we called the rest to join us there.

(Position 13): When dark clouds filled the sky * they left the fields for home.

(Position 17): That the bears would come back next spring * we teared was true.

The sentences were ordered randomly subject to the constraint that. starting with the tirst. each block of four sentences contained one sentence of each "type." The sentences were then recorded on a Sony TC- 630 recorder in a female voice at an average rate of a little more than 3 words/sec, with normal intonation but without a pause between words. Three copies of the tape were made, and on a second channel of each clicks were recorded so that, for a given sentence, the click coincided with the grammatical break on one tape, occurred two positions before the break on another, and two positions after the break on the third. The number of times the click came before, after. or within the break was the same for each "type" of sentence on all tapes. 
The positioning of the click was done while the tapes were being played back at one-quarter the speed at which the sentences had been recorded. During recording, the click was a $250-\mathrm{Hz}$ signal of $7.5 \mathrm{msec}$ duration with a 5 - $\mathrm{msec}$ rise/fall time. When the tape was played back at the normal speed for the sentences, the click was equivalent to a $1,000-\mathrm{Hz}$ signal of approximately $2 \mathrm{msec}$ duration, and of an intensity approximately that of the average level of the speech signal as judged by the VU meters.

Response booklets were prepared showing each sentence on a separate page. interleaved with blank pages.

\section{Pracedure}

The stimuli were presented through two speakers placed directly in front of the subject. the sentence coming from the right one, the click from the left. The subject's task was to listen to the stimuli, repeat back the sentence. then turn the page of the response booklet to the printed version of the sentence and mark the location of the click. The subject indicated his judgment of the click's position with an X and. when unsure. marked other likely locations with vertical slashes. To clarify what was expected, the subject was shown some fictitious samples of performance. where sometimes only one location had been checked. sometimes more than one. and the locations checked were between as well as within words. The experiment began with four practice trials to acquaint the subject with the procedure.

\section{Subjects}

The subjects were recruited from introductory psychology courses at the University of Guelph and were paid for their participation. Five male and five female subjects were assigned to each tape. making a total of 30 subjects in all.

\section{RESULTS}

When a subject made more than one response, only the first (i.e.. the subject's "first choice") was taken as

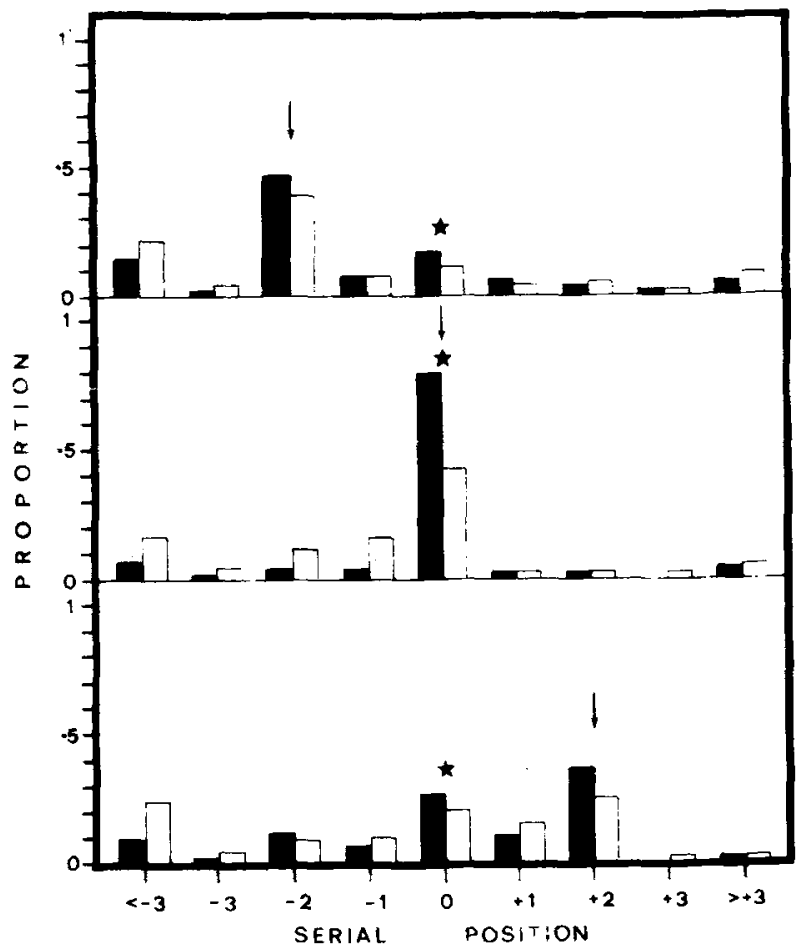

Figure 1. Distribution of responses when the click was after (top panel), coincident with (middle pane), or before (bottom panel) the grammatical break. Filled bars indicate "certain" responses, unfilled bars "uncertain" responses. The true location of the click is marked by a star, the grammatical break by an arrow.

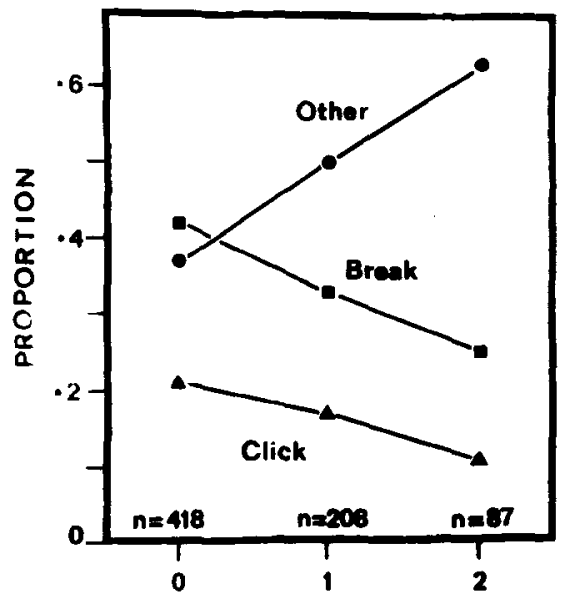

RESPONSE UNCERTAINTY

Figure 2. Proportion of occasions when the click was judged to be in the grammatical break ("Break"), in its true location ("Click"), or elsewhere ("Other"), as a function of the uncertainty of the response, infer red from the number of additional locations $(0,1$, or 2$)$ that were selected. Occasions when the click and break coincided are excluded. The ns give the number of cases for each level of "uncertainty."

his judgment of the true location of the click. In what follows. the term "response" is restricted to these. The number of additional locations checked by a subject served to characterize his response as being "certain" (when no additional locations were checked) or "uncertain" (when one or more additional locations were checked). On this criterion, $61 \%$ of all responses were "certain."

Figure 1 provides a summary of the results and displays with varying degrees of perspicuousness the findings that will be examined in greater detail below, namely: (i) "certain" responses were more likely to be correct than "uncertain" responses; (ii) incorrect "certain" responses were more likely to be in the grammatical break than were incorrect "uncertain" responses; (iii) the probability of correctly locating the click was greater when it was actually in the break; (iv) there was a tendency to prepose the click, but directional bias was minor compared to the effect of the grammatical break. The "click effect" is also clear: there were more placements in the grammatical break than in any other position.

\section{In-Break Responses and Uncertainty}

Ordinarily, one would expect a negative relationship between the correctness of a response and its uncertainty. Figure 2 shows that the proportion of correct responses declines with increasing response uncertainty, the latter being inferred from the number of additional locations checked by the subject. The proportions plotted in Figure 2 were based on pooled data from all subjects for trials when the click did not coincide with the grammatical break. Since there were only five instances of more than three additional positions being checked, these were excluded from consideration. 


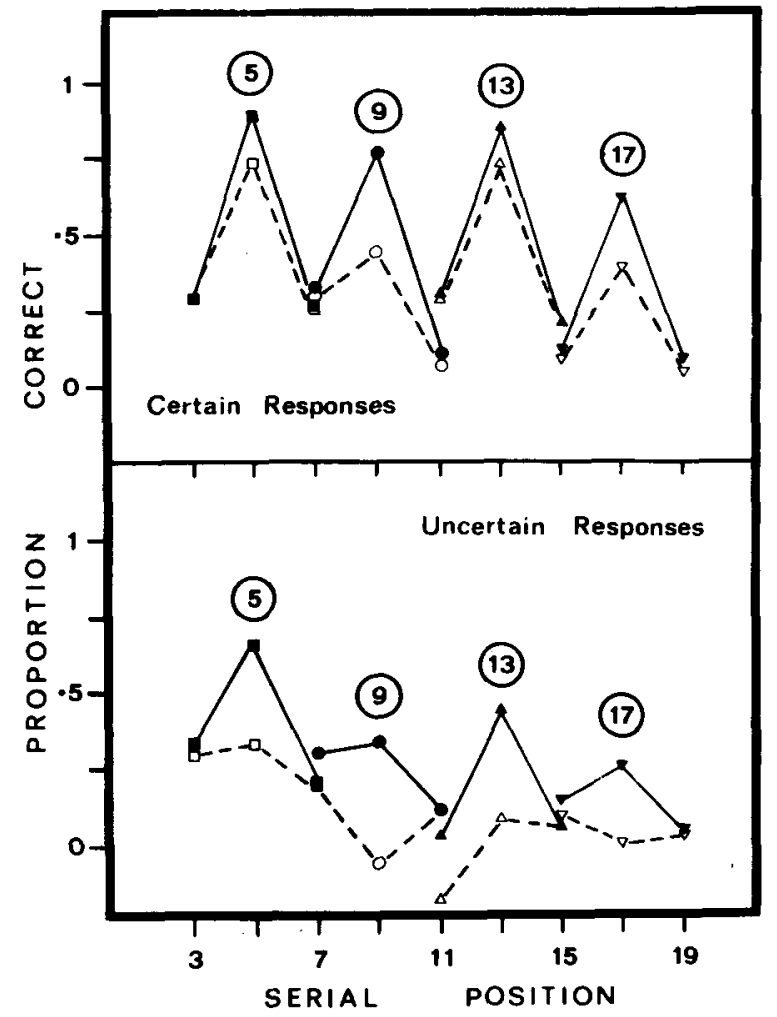

Figure 3. Variation in the proportion of correct responses with the true location of the click, when the grammatical break was in Positions 5, 9, 13, or 17 in the sentence. Unflled points indkate values obtained after the original values (filled points) were adjusted for possible response bias.

Figure 2 also shows that the greater the uncertainty of an erroneous response, the less was the likelihood that it was in the break. Although the linearity of the function is probably fortuitous, it provided a convenient strategy for testing the statistical signiticance of the drop in proportions as uncertainty increased. The value of chi square measuring differences accounted for by linearity was 10.23 $(p<.01)$, and that measuring departure from linearity was $0.02(\mathrm{p}>.75)$.

Since about a third of the subjects gave "certain" responses on more than $80 \%$ of the trials, not all subjects contributed equally to the proportions tested in the preceding analyses. Analysis by subjects would have been more appropriate, but very low ns in many cells precluded this. As a check on the possibility that the results displayed in Figure 2 are artifactual, reflecting differences between subjects and not intrasubject variation associated with uncertainty, a second analysis was carried out. Two categories of "certainty" were examined: "certain" (i.e., only one position marked) and "uncertain" (i.e., one or more additional positions marked). The comparison of interest was that between the proportion of "certain" responses located in the break and the proportion of "uncertain" responses located in the break. To ensure some degree of reliability of individual proportions, only those 14 subjects were included for whom not more than $80 \%$ or less than $20 \%$ of the responses were "certain." Cramer's procedure (cf. Fleiss, 1973, Chap. 10) was used. Chi square for homogeneity of the subjects' proportions was 9.27 ( $\mathrm{df}=13, \mathrm{p}>.35$ ). Chi square comparing the proportion of placements in the break for "certain" and "uncertain" responses (.53 and .33, respectively) was 14.56 ( $\mathrm{df}=1$, $\mathrm{p}<.001)$. These results are consistent with those reported above. Fewer in-break responses were made when the subject was less certain.

It is also informative to examine the data in terms of differences in uncertainty conditional on performance. For pooled data on all cases where the objective locations of the click and the grammatical break did not coincide, "certain" responses accounted for $66 \%$ of all correct responses, $65 \%$ of all erroneous responses placed in the break, but only $49 \%$ of all other erroneous responses. The difference between these values is significant, $\chi^{2}(2)=21.13$, $\mathrm{p}<.001$. Partitioning (cf. Fleiss, 1973, Chap. 9) gave a chi square of $0.06(p<.75)$, for the comparison between the proportions of erroneous in-break and correct responses that were "certain," and a chi square of $21.08(p<.001)$ for the difference between these taken together and the proportion of erroneous responses not in the break that were "certain." These findings suggest that subjects were as certain of erroneous in-break responses as they were of correct responses, and they were less certain when they made other erroneous responses.

\section{Incidence of Correct Responses}

Figure 3 shows how the proportion of correct responses varied with the true location of the click. In addition to our earlier observation that "certain" responses were more likely to be correct than "uncertain" responses, we see here the decided superiority of performance when the click was actually in the grammatical break, though the effect is less pronounced for "uncertain" responses.

Figure 3 also shows the results of adjusting the data for possible "response bias." The adjusted value in each case was (unadjusted value $\left.-p_{b}\right) /\left(1-p_{b}\right)$, where $\mathrm{p}_{\mathrm{b}}$, the probability of responding through response bias, was taken to be the probability of erroneously locating the click in that position, for sentences with identically located grammatical breaks. Table 1 provides a summary of these data. The adjusted

Table 1

Proportions of Correct Responses, Unadjusted and Adjusted for Response Bias

"Certain" "Uncertain"

Unadjusted Adjusted Unadjusted Adjusted

\begin{tabular}{lcccc} 
& \multicolumn{4}{c}{ Unadjusted } \\
\hline Click in break & .79 & .56 & .42 & .09 \\
Click not in break & .21 & .19 & .15 & .10 \\
\hline
\end{tabular}


values for the four cases where click and break concide (Positions 5. 9, 13, and 17) were compared statistically with the adjusted values for cases where the click was not objectively in the break. The Wilcoxon rank sum test showed the difference to be signiticant beyond the .01 level for "certain" responses. but not significant $(p>.10)$ for "uncertain" responses. The results of these analyses support the conclusion that the click was more accurately located when it had occurred in the grammatical break. and this effect is not attributable solely to response bias, at least not in the case of "certain" responses.

As a check on the possibility of an artifact due to unequal contribution of subjects to the estimates shown in Table 1, the unadjusted proportions were computed for each of 13 subjects and then averaged. The subjects selected were. those with no less than $20 \%$ and no more than $80 \%$ "certain" responses on trials when the click and break coincided. as well as on trials when they did not. For this group, the proportion of correct "certain" responses was .81 when the click had been in the break. and .16 when the click and break did not coincide. Corresponding proportions for "uncertain" responses were .41 and .15. respectively. These values were close enough to those in Table 1 to provide reassurance that the variation among the latter is not a spurious outcome of pooling.

Finally, a trend will be seen towards poorer performance when the click occurs later in the sentence. Reber (1973b) maintains that clicks occurring earlier in the sentence have a higher error rate. and that this "conforms to simple short-term memory notions."

\section{Left/Right Bias in Responding}

Figure 4 presents data on the subjects' tendency to locate the click earlier ("error to the left") or later ("error to the right") than it actually was. The difference between the proportion of errors to the right and errors to the left provides a convenient index of directional bias. As would be expected from the decreasing opportunity for errors to the right as the click position shifts to the right, the proportion of errors to the right decreases as the true position of the click moves towards the end of the sentence. There is, however, an overall tendency for errors to be preposed, particularly in the case of "uncertain" responses. Changes with practice were not systematic.

It would be a mistake to give much importance to these data, for many of the values plotted as quite unreliable. This is particularly true of those for cases where the click and break coincided, for which there were generally few errors. However, it is necessary to consider this aspect of performance in view of the significance that has been attached to the left/right biases in responding (Reber, 1973a). It is enough for

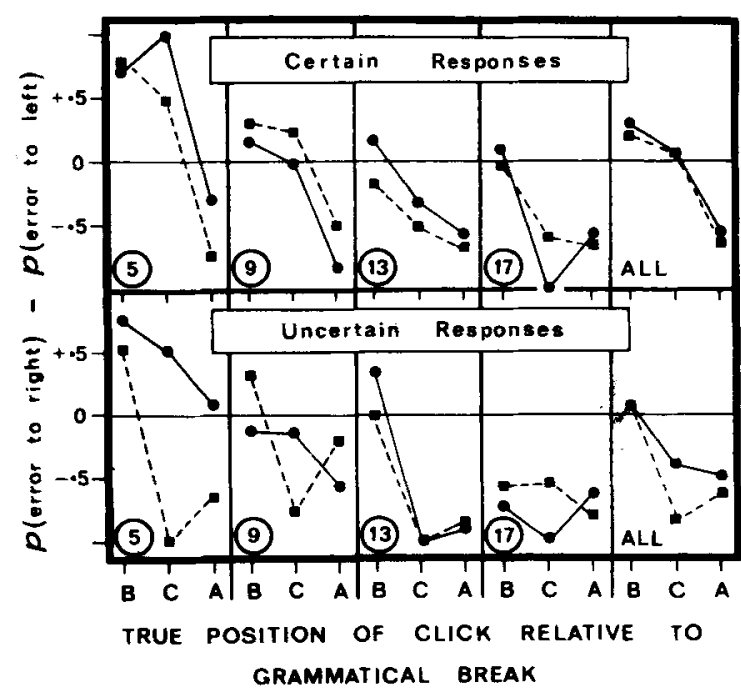

Figure 4. Difference between the proportions of pre- and postposed responses when the grammatical break was in Positions 5 , 9,13 , or 17 , as a function of whether the click was after $(A)$, before (B), or coincident with (C) the break. The solid line gives performance during the first half of the experiment, the broken line the last half.

our purposes to note that, whatever the directional bias, the effect of the grammatical break is clear. especially for "certain" responses. For these, the proportion of errors to the right is greater when the click precedes the break than when it follows. regardless of the serial position of the click. Further. the overall proportion of errors to the right was .64 when the click came before the break and $86 \%$ of these were responses in the break position. The overall proportion of errors to the left when the break came before the click was $.81,77 \%$ of which were responses in the break position.

\section{Imperfect Recall}

The analyses and results reported above took no account of whether or not the subject was able to call back the sentence without error. Recall was imperfect on approximately $8 \%$ of all trials. For these cases. when the click had been in the break, $65 \%$ of the "certain" and $38 \%$ of the "uncertain" responses were correct. When the click and break did not coincide, the corresponding values were .23 and .15 . respectively. Approximately $26 \%$ of the erroneous "certain" responses and $20 \%$ of the erroneous "uncertain" responses were in the grammatical break. Given the low incidence of imperfect recall and similar trends in the data, the effect of including these responses in the other analyses must be negligible.

\section{DISCUSSION}

In this investigation, the number of locations checked by the subject was taken as an indication of 
the certainty of his judgment. The fact that responses classified as "certain" on this basis were correct more often than those classified as "uncertain," may be regarded as a sign of the validity of the criterion. Granted this, we may next conclude from the data that the less certain the subjects were about the correct location of the click, the less likely they were to judge the click as having been in the grammatical break. Since it is reasonable to expect response biases to have operated more freely when the subjects were less certain, these results argue against attributing responses in the break entirely to a bias in responding induced by the position of the grammatical break. Rather, it appears that such responses reflect the subject's perception of when the click had actually occurred. And while the response must be considered an error when the click had actually occurred elsewhere, it is not necessarily an error in the subject's reporting of what he had actually perceived. If an erroneous perception, it may be no less "perceptual" than a veridical perception of the click's location. The observation that erroneous responses in the break were as likely as correct responses to be "certain," and more likely than other erroneous responses, is consistent with this.

Even if we were to conclude that erroneous in-break responses were solely the result of response bias, the higher incidence of correct responding when the break and the click coincided must be interpreted otherwise. We have seen that adjustment of the probability of a correct response to allow for response bias did not eliminate the superior performance when the click had been in the break, even though this adjustment required treating all erroneous in-break responses as the effect of response bias.

The general conclusion from these observations is that the "click effect" is not a consequence of a bias in responding induced by the position of the grammatical break. Rather, it appears that the effect derives from the manner in which sentences are processed during presentation.

\section{REFERENCES}

Abrams, K., \& Bever, T. G. Syntactic structure modifies attention during speech perception and recognition. Quarterly Journal of Experimental Psychology, 1969, 21, 280-290.

Bever, T. G. Serial position and response biases do not account for the effect of syntactic structure on the location of brief noises during sentences. Jourmal of Psycholinguistic Research, 1973, 2, 287.288.

Bever. T. G., Lackner. J., \& Kirk, R. The underlying structures of sentences are the primary units of immediate speech processing. Perception \& Psychophysics, 1969, 5. 225-234.

FleISS, J. L. Statistical methods for rates and proportions. New York: Wiley, 1973.

Fodor, J. A., \& BEver, T. G. The psych, ical reality of linguistic segments. Joumal of Verbal Learning and Verbal Behavior. 1965, 4, 414-420.

FODOR, J. A., BEVER, T. G., \& GARRETT, M. F. The psychology of language. New York: McGraw-Hill, 1974.

Garrett, M., Bever, T. G.. \& Fodor, J. A. The active use of grammar in speech perception. Perception \& Psychophysics, $1966,1,30-32$.

LADEFOGED, P. Three areas of experimental phonetics. London: Oxford University Press, 1967.

REBER, A. S. Locating clicks in sentences: Left, center, and right. Perception \& Psychophysics, 1973, 13. 133-138. (a)

REBER, A. S. What clicks may tell us about speech perception. Journal of Psycholinguistic Research, 1973, 2, 286-287. (b)

Reber, A. S., \& ANDERSON, J. R. The perception of clicks in linguistic and nonlinguistic messages. Perception \& Psychophysics, 1970, 8, 81-89.

\section{NOTE}

1. Let $p$ '. be the probability of a "true" correct response, $p_{b}$ the probability of a correct response through response bias, and $p_{c}$ the observed probability of a correct response. When $p_{b}>0, p_{c}$ will provide an inflated estimate of $p^{\prime}{ }^{.}$. This is because when a "true" correct response is absent (which happens with probability $\left.1-p^{\prime}\right)$, there is still the probability $\left(p_{b}\right)$ that a correct response occurs through response bias. This leads to $\mathrm{p}_{\mathrm{c}}=\mathrm{p}_{\mathrm{i}}{ }+$ $\left(1-p^{\prime}\right) p_{b}$. Whence we have $p_{i}^{\prime}=\left(p_{t}-p_{b}\right) /\left(1-p_{b}\right)$.

(Received for publication September 2. 1975; revision received January $19,1976$. 